\title{
What factors are associated with frequent unhealthy snack-food consumption among Australian secondary-school students?
}

\author{
Philippa Niven ${ }^{1}$, Maree Scully ${ }^{1}$, Belinda Morley ${ }^{1, *}$, Louise Baur ${ }^{2}$, David Crawford ${ }^{3}$, \\ lain S Pratt ${ }^{4}$ and Melanie Wakefield ${ }^{1}$ for the NaSSDA Study Team $†$ \\ ${ }^{1}$ Centre for Behavioural Research in Cancer, Cancer Council Victoria, 615 St Kilda Road, Melbourne, VIC 3004, \\ Australia: ${ }^{2}$ Discipline of Paediatrics \& Child Health, The University of Sydney, Sydney, Australia: ${ }^{3}$ Centre for Physical \\ Activity and Nutrition Research, Deakin University, Melbourne, Australia: ${ }^{4}$ Education and Research, Cancer Council \\ Western Australia, Perth, Australia
}

Submitted 6 July 2014: Final revision received 13 October 2014: Accepted 15 October 2014: First published online 2 December 2014

\begin{abstract}
Objective: To examine demographic and behavioural correlates of unhealthy snack-food consumption among Australian secondary-school students and the association between their perceptions of availability, convenience and intake with consumption.

Design: Cross-sectional survey of students' eating, physical activity and sedentary behaviours using validated instruments administered via an online questionnaire. Setting: Australian secondary schools across all states/territories.

Subjects: Secondary-school students aged 12-17 years participating in the 2009-10 National Secondary Students' Diet and Activity (NaSSDA) survey ( $n$ 12 188).

Results: Approximately one in five students (21\%) reported consuming unhealthy snack foods $\geq 14$ times/week ('frequent snackers'). After adjusting for all covariates, older students and those with a BMI of $\geq 25 \mathrm{~kg} / \mathrm{m}^{2}$ were less likely to be frequent snackers, while students who reported high fast-food and high sugarsweetened beverage consumption and those who watched television for $>2 \mathrm{~h} / \mathrm{d}$ were more likely to snack frequently. Furthermore, after adjusting for all covariates and demographic factors, students who agreed that snack foods are usually available at home, convenient to buy and that they eat too many snack foods were more likely to be snacking frequently. Conversely, students who agreed that fruit is a convenient snack were less likely to be frequent snackers.

Conclusions: Frequent unhealthy snack-food consumption appears to cluster with other poor health behaviours. Perceptions of availability and convenience are factors most readily amenable to change, and findings suggest interventions should focus on decreasing the availability of unhealthy snack foods in the home and promoting healthier options such as fruit as convenient snacks.
\end{abstract}

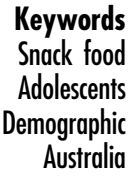

Keywords Adolescents Australia
Snack foods are generally energy-dense and nutrient-poor and are commonly consumed by adolescents, especially in their leisure time ${ }^{(1)}$. Ninety per cent of Australian secondary-school students report consuming extra or non-essential foods on a daily basis, with energy-dense snacks comprising the greatest proportion of the extra foods consumed ${ }^{(2)}$. Research suggests that intake of extra foods and an energy-dense diet may displace the consumption of core foods such as fruit and vegetables $^{(3,4)}$, and energy-dense, high-fat diets have been

$\uparrow$ The NaSSDA Study Team comprises Cancer Council Victoria: Belinda Morley, Maree Scully, Melanie Wakefield; Technical Advisory Group: Louise Baur (Chair), Anthony Okely, Iain S. Pratt, Jane Bowen, Jo Salmon, Victoria Flood, David Crawford, Anthony Worsley. identified as risk factors in the development of obesity during childhood and adolescence ${ }^{(5)}$.

Despite the health risks associated with frequent consumption of unhealthy snack foods, literature assessing the demographic and behavioural correlates of adolescent snacking is limited. Given that studies use different definitions of snack foods, it is difficult to determine just how frequent snacking is for this age group ${ }^{(6,7)}$. Some studies define snack foods as anything consumed between main meals (core or non-core food items), while other studies focus on individual snack-food items or only on snacks consumed at a specific time of day. Snacking habits in relation to frequency, size and energy content of snacks also differ across countries ${ }^{(8)}$, making it difficult 
to compare studies conducted overseas with those undertaken in Australia.

In line with the differing methodologies, results assessing demographic differences in snack-food consumption are inconsistent. Most Australian studies have found no sex difference in adolescent snack-food consumption ${ }^{(2,9,10)}$; however, other studies conducted in Australia and internationally suggest that boys are consuming more snacks ${ }^{(11,12)}$ and high-fat foods ${ }^{(13)}$ than are girls, whereas a Swedish study found girls to be consuming more in between meals than boys ${ }^{(14)}$. While two Australian studies have found that snacking increases with age ${ }^{(10,15)}$, some contradictory results have also been reported. Specifically, Cameron et al. ${ }^{(16)}$ found that snack consumption was similar for 4- to 16-yearolds, while a longitudinal study found snacking actually decreased with age ${ }^{(9)}$. A review by Larson and Story ${ }^{(17)}$ indicated that the majority of studies either showed no evidence of a relationship between snacking and weight status or found that those who consumed more snacks were less likely to be obese. Gregori et $a l^{(6)}$ also noted that the use of different definitions of snack foods, and the different BMI profiles of participants in the studies reviewed, makes it difficult to conclude what role snacking has in relation to obesity and thus why the literature is equivocal.

Studies examining the impact of socio-economic position (SEP) on snack-food intake also differ on the measure of snack foods applied and on the measure of SEP employed (area-based, parental education or income). Despite this, studies have generally found that low SEP adolescents are likely to consume more snacks ${ }^{(18)}$, especially those which are fat-dense ${ }^{(19)}$, and have a higher intake of dietary fat in general ${ }^{(13,20,21)}$ compared with adolescents from relatively higher SEP groups. In contrast, an Australian state-based school survey that used an area-based measure of SEP reported no association between socio-economic background and the consumption of specific snack foods ${ }^{(22)}$.

There is consistent evidence to show that consumption of snack foods is positively associated with television viewing ${ }^{(20,23-30)}$, yet research assessing the relationship between snacking and other eating, physical activity and sedentary behaviours and sleep duration is scarce. A small number of studies have looked at the association between snack consumption and fruit and vegetable intake in adolescents. Lazzeri et $a l .{ }^{(31)}$ reported a significant association between irregular snack consumption (defined as not having snacks both mid-morning and afternoon) and low frequency of fruit intake but no significant difference for vegetable consumption. Sebastian et al. ${ }^{(32)}$ found a similar association, reporting that fruit intake increased with higher snacking frequency for both adolescent boys and girls but did not differ for vegetable consumption. In regard to the influence of sleep duration on snacking, Weiss et al. ${ }^{(33)}$ found that shorter sleep duration was associated with high energy intake from snacks in unadjusted analyses; however, this association was attenuated and no longer statistically significant after adjusting for participant characteristics.
Other studies have found a similar negative association between sleep and snack-food consumption in adults ${ }^{(34-36)}$. To our knowledge, no studies have assessed the relationship between adolescent snacking and physical activity and non-core dietary intake.

Adolescents' perceptions of the availability and convenience of foods could also influence their dietary intake. Snack foods are commonly perceived to be available within adolescents' home environments ${ }^{(37,38)}$ and the availability of unhealthy foods at home appears to be a robust predictor of consumption of obesity-promoting foods and drinks ${ }^{(11)}$. Pearson et al. ${ }^{(39)}$ and Cutler et al. ${ }^{(40)}$ found similar results, reporting that home availability of snack foods was positively associated with energy-dense snack consumption. Adolescents perceive the inconvenience of food options to be a barrier to healthy eating ${ }^{(41,42)}$ and sweet and salty snack foods are attractive options because they require little or no preparation ${ }^{(43,44)}$. Therefore, even if foods are readily available to adolescents, if they are perceived to be inconvenient they are less likely to be consumed.

The aim of the present study was to identify what factors are associated with frequent consumption of snack foods among Australian secondary-school students. Specifically, we aimed to explore whether there are demographic and behavioural differences in students' frequency of consumption of snack foods and whether individual perceptions of availability, convenience and intake are associated with their consumption.

\section{Methods}

\section{Design and procedure}

Data were obtained from students participating in the 2009-10 National Secondary Students' Diet and Activity (NaSSDA) survey (school response rate: 39\%; student response rate: $54 \%$ ). The cross-sectional survey collected data from students in Years 8 to 11 (ages 12 to 17 years) from a representative sample of Australian schools. A full description of the methods has been reported elsewhere ${ }^{(45)}$. In brief, the sampling procedure was a stratified two-stage probability design, with schools randomly selected at the first stage of sampling and classes selected within schools at the second stage. Students completed a web-based questionnaire assessing their eating, physical activity and sedentary behaviours as well as their intake and perceptions of snack foods. Students also had height and weight measured by trained researchers in a confidential setting. Informed written parent/carer and student consent was required for participation in each component of the study.

\section{Measures}

\section{Demographics}

Information on students' sex, school year level and postcode was recorded. Residential postcode was used to 
classify students into low, medium or high SEP according to the Socio-Economic Index for Areas Index of Relative Socioeconomic Disadvantage ${ }^{(46)}$ and into metropolitan or rural/ regional geographic location according to the Rural, Remote and Metropolitan Areas classification ${ }^{(47)}$. Students' height and weight were taken in accordance with standardised protocols $^{(48)}$ and used to calculate BMI (weight/height ${ }^{2}$ ). Students were classified as either healthy weight/underweight or overweight/obese according to internationally recognised cut-offs developed for children and adolescents ${ }^{(49)}$. Students' weekly spending money was also assessed.

\section{Behaviours}

Measures used to assess students' core and non-core food consumption, physical activity and sleeping patterns have been reported in greater detail elsewhere ${ }^{(45)}$. In summary, core and non-core food consumption was assessed using short dietary questions developed by the NSW Centre for Public Health Nutrition ${ }^{(50)}$. Students were asked how many servings of vegetables and fruit they usually eat each day and how often they consume sugary drinks and meals or snacks from fast-food or takeawayfood places. Physical activity was assessed using the 60 min moderate-to-vigorous physical activity screening measure, which is reliable, valid and correlates well with objective measures of physical activity ${ }^{(51)}$. Sleeping patterns were assessed by asking students to record the time they went to bed and turned the light out the previous night and the time they woke up that morning; or, if either time was not 'usual', the time they usually went to bed on a school night or woke up on a school day. To assess television viewing, students were asked to indicate how long they spent watching commercial television on a typical school day, Saturday and Sunday. A weighted average daily time spent watching commercial television was calculated and dichotomised to indicate those watching for $>2 \mathrm{~h} / \mathrm{d}$ (high viewers).

\section{Snack-food consumption}

Students were asked to indicate how often they consume: (i) ice cream, icy poles or ice blocks; (ii) potato crisps/ chips or other salty snacks (such as Twisties ${ }^{\mathrm{TM}}$, corn chips); (iii) confectionery (such as lollies and chocolates); and (iv) sweet foods (such as sweet biscuits, cakes or muffins). Response options included 'never', 'less than once a week', 'about 1-2 times a week', 'about 3-4 times a week', 'about 5-6 times a week', 'about once a day' and '2 or more times a day'. As per previous studies ${ }^{(52-54)}$, we assigned each response option a weekly equivalent value and then summed students' responses for the four snackfood items (range 0-56 times/week). Given the Australian Dietary Guidelines ${ }^{(55)}$ recommend limiting foods containing saturated fat, added salt and added sugars, we chose to classify 'frequent snackers' as those with a score of $\geq 14$ times/week (equivalent to snacking two or more times daily on average).
Perceptions of snack foods

Students were asked to indicate the extent to which they agreed or disagreed with the following six statements relating to their perceptions of snack foods: (i) 'Snack foods are usually available in my home'; (ii) 'Snack foods are convenient to buy'; (iii) 'I eat too many snack foods'; (iv) 'It is easy to find healthy snacks (e.g. fruit, yoghurt) in my home'; (v) 'Fruit is a convenient snack'; and (vi) 'I eat enough fruit'. For each question, five-point response scales ranged from 'strongly disagree' to 'strongly agree'. Responses were dichotomised to indicate those who agreed $v$. those who disagreed or neither agreed nor disagreed.

\section{Statistical analyses}

Data analyses were conducted using the statistical software package Stata SE 12.1. Data were weighted by state, education sector, school year and sex to ensure the sample obtained reflected the population distribution ${ }^{(56)}$. All analyses accounted for the complex survey design by using the svy: prefix command in Stata SE 12.1. Two multivariable logistic regression models were analysed. The first model tested the association between frequent consumption of unhealthy snacks and demographic and behavioural factors. The second model tested the association between frequent consumption and students' perceptions of availability, convenience and intake of unhealthy snack foods. Both models controlled for all covariates, school-level clustering and school type and the second model also controlled for demographic characteristics. Due to the large sample size, a conservative significance level of $P<0.01$ was applied.

\section{Results}

As shown in Table 1, overall, 53\% of students in our sample were male and there was a slightly higher proportion of students in Years $8(31 \%)$ and $9(27 \%)$ compared with the older year levels $(22 \%$ in Year 10 and $20 \%$ in Year 11). Thirty-two per cent of students were categorised as low SEP, $42 \%$ as medium SEP and $26 \%$ as high SEP (compared with 33\% low, $42 \%$ medium and $26 \%$ high SEP for the Australian population $)^{(57)}$. The majority of the students were classified as not overweight or obese (77\%) and resided in a metropolitan location (64\%). Approximately one-quarter of students (27\%) had less than SAUD 10 disposable income each week while almost one-third (31\%) had SAUD 40 or more.

Overall, $21 \%$ of students were classified as 'frequent snackers', reporting they consume ice cream, potato crisps/chips, confectionery and/or sweet foods fourteen or more times per week. As shown in Table 2, frequent snacking differed significantly by year level, BMI, fast-food and sugar-sweetened beverage consumption and daily television viewing. Students in Year 11 and those who 
Table 1 Characteristics of the sample: secondary-school students aged 12-17 years participating in the 2009-10 National Secondary Students' Diet and Activity (NaSSDA) survey ( $n$ 12 188), Australia

\begin{tabular}{|c|c|c|}
\hline & $n$ & $\%$ \\
\hline \multicolumn{3}{|l|}{ Sex } \\
\hline Male & 6460 & $53 \cdot 0$ \\
\hline Female & 5728 & $47 \cdot 0$ \\
\hline \multicolumn{3}{|l|}{ Year level } \\
\hline 8 & 3772 & 31.0 \\
\hline 9 & 3340 & $27 \cdot 4$ \\
\hline 10 & 2647 & $21 \cdot 7$ \\
\hline 11 & 2429 & $19 \cdot 9$ \\
\hline \multicolumn{3}{|l|}{ BMI category } \\
\hline Healthy weight/underweight & 9418 & $77 \cdot 3$ \\
\hline Overweight/obese & 2770 & $22 \cdot 7$ \\
\hline \multicolumn{3}{|l|}{ Socio-economic position } \\
\hline Low & 3932 & $32 \cdot 3$ \\
\hline Medium & 5062 & 41.5 \\
\hline High & 3194 & $26 \cdot 2$ \\
\hline \multicolumn{3}{|l|}{ Geographic location } \\
\hline Metropolitan & 7841 & 64.3 \\
\hline Rural/regional & 4347 & $35 \cdot 7$ \\
\hline \multicolumn{3}{|l|}{ Weekly spending money } \\
\hline$<$ SAUD 10 & 3262 & $26 \cdot 8$ \\
\hline \$AUD 10-39 & 5156 & $42 \cdot 3$ \\
\hline$\geq \$$ AUD 40 & 3769 & $30 \cdot 9$ \\
\hline
\end{tabular}

Based on unweighted data.

Percentages are rounded so may not sum to $100 \%$.

were classified as overweight or obese were significantly less likely to be frequent snackers compared with Year 8 students and those not overweight or obese, respectively. On the other hand, students who reported consuming fast food at least weekly and 1-2 cups of sugar-sweetened beverages each day, as well as those who reported watching commercial television for $>2 \mathrm{~h} / \mathrm{d}$, were significantly more likely to be frequent snackers.

As Table 3 highlights, students who agreed that snack foods are usually available at home, are convenient to buy and that they eat too many snack foods were significantly more likely to be frequent snackers. In contrast, those who agreed that fruit is a convenient snack were significantly less likely to be classified as frequent snackers. Snacking frequency was unrelated to students' perceived ease of finding healthy snacks at home or whether they believed they eat enough fruit.

\section{Discussion}

These results indicate that frequent snack-food consumption among Australian secondary-school students appears to be similar across demographic groups, with the exception of year level and BMI. Further, frequent snacking was associated with high fast-food and high sugar-sweetened beverage consumption and with watching commercial television for $>2 \mathrm{~h} / \mathrm{d}$. To the best of our knowledge, the present study is the first one to show that frequent snack-food consumption clusters with other poor eating behaviours among adolescents.
Our results indicate a trend for frequent snack-food consumption to decline with increasing year level. This pattern is consistent with an earlier Australian cohort study which found that snacking in general decreased with increasing age ${ }^{(9)}$. However, a more recent Australian crosssectional study suggested that consumption of energydense snacks was relatively similar in children aged 4 to 16 years ${ }^{(16)}$. The smaller sample size and broader age groups assessed in the latter study may partly explain these different findings. The lower prevalence of frequent snacking among Year 11 students in our study could be due to this group consuming more snacks from fast-food outlets.

We also found that overweight/obese adolescents were less likely to be frequent snackers, as has been reported by many other studies ${ }^{(17)}$. It is possible that dieting (not assessed in the current study) is a mediating factor between BMI and snacking. Overweight or obese adolescents may be restricting their intake of snack foods in an attempt to lose weight. Howe et al. ${ }^{(58)}$ found that there was a significant interaction between New Zealand adolescents' treat food scores and dieting status, suggesting there was a positive association between treat food scores and BMI only among non-dieters. There is also evidence from the USA which indicates that overweight and obese respondents may underestimate their consumption in self-report ${ }^{(59,60)}$.

In line with other Australian studies, we found no significant difference in adolescents' frequent snack-food consumption by sex ${ }^{(2,9,10)}$ or area-based $\mathrm{SEP}^{(22)}$. Available weekly spending money, which is likely to be higher among more advantaged students, was also unrelated to snacking frequency. However, given there is some evidence of social disparities in snack-food consumption among Scottish schoolchildren ${ }^{(18,19)}$, it would be beneficial for future research to validate our findings using a range of individual-level SEP measures (e.g. household income and parental education). Inclusion of parental nutrition knowledge as an additional measure of SEP may be important as there is evidence from the USA that the majority of adolescent snacking occurs within the home environment ${ }^{(61)}$.

We found that frequent consumption of unhealthy snack foods was more common among adolescents who reported high fast-food and high sugar-sweetened beverage consumption. This clustering of poor eating behaviours highlights the need for interventions to take a whole-of-diet approach rather than focusing on reducing snack-food consumption alone. Interestingly, frequent snacking was unrelated to low intake of fruit or vegetables. This is contrary to what we would expect if energy-dense foods were to displace the consumption of healthier foods ${ }^{(3,4)}$. This lack of association may indicate that snack foods are being consumed in addition to, rather than instead of, fruit and vegetables. This assumption is also supported by the fact we found no association between frequent snacking and students' perceptions that they eat enough fruit. 
Table 2 Results from logistic regression analyses examining demographic and behavioural predictors of snack-food consumption among secondary-school students aged 12-17 years participating in the 2009-10 National Secondary Students' Diet and Activity (NaSSDA) survey $(n 11971)^{\star}$, Australia

\begin{tabular}{|c|c|c|c|c|}
\hline \multirow[b]{3}{*}{ Covariate } & \multicolumn{4}{|c|}{ Snack-food consumption ( $\geq 14$ times/week) } \\
\hline & \multicolumn{4}{|c|}{ Multivariable model† } \\
\hline & Adjusted \% & Adjusted OR & $95 \% \mathrm{Cl}$ & $P$ value \\
\hline \multicolumn{5}{|l|}{ Demographics } \\
\hline Sex & & & & 0.010 \\
\hline Male & $19 \cdot 3$ & 1.00 & Ref. & \\
\hline Female & $22 \cdot 0$ & 1.20 & $1.05,1.38$ & \\
\hline Year level & & & & 0.006 \\
\hline 8 & $22 \cdot 5$ & 1.00 & Ref. & \\
\hline 9 & $22 \cdot 1$ & 0.97 & $0.84,1.13$ & \\
\hline 10 & $19 \cdot 5$ & 0.81 & $0.68,0.97$ & \\
\hline 11 & $17 \cdot 8$ & 0.72 & $0.59,0.87$ & \\
\hline BMI category & & & & $<0.001$ \\
\hline Healthy weight/underweight & $22 \cdot 3$ & 1.00 & Ref. & \\
\hline Overweight/obese & 14.7 & 0.57 & $0.49,0.67$ & \\
\hline Socio-economic position & & & & 0.824 \\
\hline Low & $20 \cdot 2$ & 1.00 & Ref. & \\
\hline Medium & $20 \cdot 5$ & 1.03 & $0.90,1.17$ & \\
\hline High & $21 \cdot 1$ & 1.06 & $0.86,1.31$ & \\
\hline Geographic location & & & & 0.159 \\
\hline Metropolitan & $19 \cdot 9$ & 1.00 & Ref. & \\
\hline Rural/regional & 21.4 & $1 \cdot 11$ & $0.96,1.29$ & \\
\hline Weekly spending money & & & & 0.970 \\
\hline$<$ SAUD 10 & 20.4 & 1.00 & Ref. & \\
\hline \$AUD 10-39 & $20 \cdot 4$ & 1.00 & $0.88,1 \cdot 15$ & \\
\hline$\geq \$ A \cup D 40$ & 20.7 & 1.02 & $0.84,1.25$ & \\
\hline \multicolumn{5}{|l|}{ Behaviours } \\
\hline Vegetable consumption & & & & 0.297 \\
\hline$\leq 1$ serving/d & $21 \cdot 2$ & 1.07 & $0.94,1.21$ & \\
\hline$\geq 2$ servings/d & $20 \cdot 2$ & 1.00 & Ref. & \\
\hline Fruit consumption & & & & 0.057 \\
\hline$\leq 1$ serving $/ d$ & $22 \cdot 0$ & $1 \cdot 15$ & $1.00,1.33$ & \\
\hline$\geq 2$ servings/d & $19 \cdot 9$ & 1.00 & Ref. & \\
\hline Fast-food consumption & & & & $<0.001$ \\
\hline$<1$ time/week & 14.5 & 1.00 & Ref. & \\
\hline$\geq 1$ time/week & $27 \cdot 7$ & $2 \cdot 37$ & $2 \cdot 08,2 \cdot 71$ & \\
\hline Sugar-sweetened beverage consumption & & & & $<0.001$ \\
\hline 4-6 cups/week or less & $17 \cdot 7$ & 1.00 & Ref. & \\
\hline $1-2$ cups/d or more & 35.9 & $2 \cdot 80$ & $2 \cdot 38,3.28$ & \\
\hline Physical activity & & & & 0.755 \\
\hline$\leq 3 \mathrm{~d} /$ week & $20 \cdot 3$ & 0.98 & $0.86,1 \cdot 12$ & \\
\hline$\geq 4 \mathrm{~d} /$ week & $20 \cdot 7$ & 1.00 & Ref. & \\
\hline Television viewing & & & & $<0.001$ \\
\hline$\leq 2 \mathrm{~h} / \mathrm{d}$ & $17 \cdot 3$ & 1.00 & Ref. & \\
\hline$>2 \mathrm{~h} / \mathrm{d}$ & $26 \cdot 5$ & 1.82 & $1 \cdot 62,2.04$ & \\
\hline Sleep & & & & 0.073 \\
\hline$<8 \mathrm{~h}$ & $22 \cdot 3$ & $1 \cdot 16$ & $0.99,1.37$ & \\
\hline$\geq 8 \mathrm{~h}$ & $20 \cdot 0$ & 1.00 & Ref. & \\
\hline
\end{tabular}

Ref., referent category.

*Sample size smaller due to missing values.

†Adjusted for all covariates, school-level clustering and school type.

Frequent snacking was not associated with low physical activity. It is possible that adolescents may consume snack foods as a treat after participating in physical activity given that unhealthy foods and beverages are commonly available $^{(62,63)}$ and sold at sporting clubs and venues ${ }^{(63,64)}$. Sporting clubs are also a location in which exposure to unhealthy food marketing via sports sponsorship is high $^{(65)}$. Food marketing has consistently been shown to influence children's food preferences, purchases and consumption $^{(66-68)}$. Therefore, adolescents participating in sport more often may have greater exposure to unhealthy food marketing and thus increased consumption of unhealthy snack foods.

Adolescents who agreed that snack foods are usually available at home were more likely to be consuming snack foods on a frequent basis. This suggests a need to reduce the availability of these unhealthy snack options and increase the availability of healthier options. However, our study also found that frequent snack-food consumption was unrelated to perceived ease of finding healthy 
Table 3 Influence of adolescents' perceptions of availability, convenience and intake on adolescents' snack-food consumption: secondary-school students aged 12-17 years participating in the 2009-10 National Secondary Students' Diet and Activity (NaSSDA) survey $(n 11923)^{*}$, Australia

\begin{tabular}{|c|c|c|c|c|}
\hline \multirow[b]{3}{*}{ Covariate } & \multicolumn{4}{|c|}{ Snack-food consumption ( $\geq 14$ times/week) } \\
\hline & \multicolumn{4}{|c|}{ Multivariable model† } \\
\hline & Adjusted \% & Adjusted OR & $95 \% \mathrm{Cl}$ & $P$ value \\
\hline Snack foods are usually available in my home & & & & $<0.001$ \\
\hline Disagree/neither agree nor disagree & 13.5 & 1.00 & Ref. & \\
\hline Agree/strongly agree & $24 \cdot 0$ & $2 \cdot 10$ & $1.83,2.41$ & \\
\hline It is easy to find healthy snacks in my home & & & & 0.010 \\
\hline Disagree/neither agree nor disagree & 22.4 & 1.00 & Ref. & \\
\hline Agree/strongly agree & $19 \cdot 9$ & 0.85 & $0.75,0.96$ & \\
\hline Snack foods are convenient to buy & & & & 0.001 \\
\hline Disagree/neither agree nor disagree & $18 \cdot 6$ & 1.00 & Ref. & \\
\hline Agree/strongly agree & $21 \cdot 8$ & $1 \cdot 24$ & $1.09,1.40$ & \\
\hline Fruit is a convenient snack & & & & $<0.001$ \\
\hline Disagree/neither agree nor disagree & $27 \cdot 4$ & 1.00 & Ref. & \\
\hline Agree/strongly agree & 18.9 & 0.59 & $0.51,0.68$ & \\
\hline I eat too many snack foods & & & & $<0.001$ \\
\hline Disagree/neither agree nor disagree & $15 \cdot 0$ & 1.00 & Ref. & \\
\hline Agree/strongly agree & 30.5 & 2.60 & $2 \cdot 29,2.94$ & \\
\hline I eat enough fruit & & & & 0.438 \\
\hline Disagree/neither agree nor disagree & $20 \cdot 2$ & 1.00 & Ref. & \\
\hline Agree/strongly agree & 20.9 & 1.04 & $0.93,1.17$ & \\
\hline
\end{tabular}

Ref., referent category.

*Sample size smaller due to missing values.

†Adjusted for demographic characteristics, all covariates, school-level clustering and school type.

snacks at home. Alternatively, we found students who agreed that fruit is a convenient snack were significantly less likely to be frequent snackers. This suggests that making healthy snacks available within the home is not sufficient to prompt behaviour change. Adolescents need to perceive these food items as convenient in order to choose the healthy option. Frequent snacking was more common among adolescents who agreed that snack foods are convenient to buy. Improving the convenience of healthy options, such as peeled and cut fruit and vegetables, at places where adolescents purchase snack foods (e.g. the school canteen and fast-food outlets) could be important in reducing unhealthy snack-food consumption.

In considering these findings, it is important to acknowledge the limitations of the present study. First, the cross-sectional study design precludes the inference of causality. Second, self-reported behaviour allows the possibility of recall and social desirability bias (e.g. underreporting of snack-food consumption). Third, given the use of an active consent procedure, the rate of student participation was low, although the response rate was comparable to similar Australian state-based school surveys ${ }^{(69,70)}$. Fourth, our measures focused on snacking frequency and did not assess the portion size or energy content of the snacks being consumed. Future research in this area should take these important snacking attributes into account. Lastly, the study employed an area-based measure of SEP. As mentioned above, future research may benefit from including individual-level measures such as parental education or nutritional knowledge. Strengths of the present study include the large representative sample and the use of student self-report rather than parent proxy report. This enabled the capture of snacking behaviour that may occur outside the home environment and out of parent control, view or knowledge.

The present study indicates that a high proportion of Australian adolescents are snacking on a frequent basis, in conflict with the new Australian Dietary Guideline recommendation that discretionary foods be limited ${ }^{(55)}$. Of particular concern, frequent snacking appears to cluster with other poor health behaviours, such as high fast-food and sugar-sweetened beverage consumption and frequent television viewing. Perceptions of availability and convenience influence frequent snack-food consumption. Given these are factors most readily amenable to change, interventions that focus on decreasing the availability of unhealthy snack foods in the home, in conjunction with promoting healthy and convenient snack options such as fruit, may prove most effective.

\section{Acknowledgements}

Acknowledgements: The authors thank the school principals, teachers and students who participated in the study. Financial support: The NaSSDA survey was jointly funded by state Cancer Councils through Cancer Council Australia; and by the National Heart Foundation of Australia. M.W. is supported by a Principal Research Fellowship form the National Health and Medical Research Council. The funders 
had no role in the design, analysis or writing of this article. Conflict of interest: None. Authorship: All authors have contributed to the present paper by being involved in conceiving and designing the study or in analysis and interpretation of data, and in writing and revising the paper. I-View was responsible for fieldwork coordination. Ethics of buman subject participation: The NaSSDA study was approved by the Human Research Ethics Committee of Cancer Council Victoria. In addition, approval was obtained from the education authority for each sector in each State and Territory, as well as the principal at each selected school.

\section{References}

1. Savige G, Macfarlane A, Ball K et al. (2007) Snacking behaviours of adolescents and their association with skipping meals. Int J Behav Nutr Phys Act 4, 36.

2. Savige GS, Ball K, Worsley A et al. (2007) Food intake patterns among Australian adolescents. Asia Pac J Clin Nutr 16, 738-747.

3. Webb KL, Lahti-Koski M, Rutishauser I et al. (2006) Consumption of 'extra' foods (energy-dense, nutrient-poor) among children aged 16-24 months from western Sydney, Australia. Public Health Nutr 9, 1035-1044.

4. Kant AK \& Graubard BI (2005) Energy density of diets reported by American adults: association with food group intake, nutrient intake, and body weight. Int J Obes (Lond) 29, 950-956.

5. Ambrosini GL, Emmett PM, Northstone K et al. (2012) Identification of a dietary pattern prospectively associated with increased adiposity during childhood and adolescence. Int J Obes (Lond) 36, 1299-1305.

6. Gregori D, Foltran F, Ghidina M et al. (2011) Understanding the influence of the snack definition on the association between snacking and obesity: a review. Int J Food Sci Nutr 62, 270-275.

7. Johnson GH \& Anderson GH (2010) Snacking definitions: impact on interpretation of the literature and dietary recommendations. Crit Rev Food Sci Nutr 50, 848-871.

8. Adair LS \& Popkin BM (2005) Are child eating patterns being transformed globally? Obes Res 13, 1281-1299.

9. Magarey A \& Boulton J (1995) The Adelaide Nutrition Study. 4. Meal habits and distribution of energy and nutrients throughout the day at ages 11, 13 and 15 years. Aust J Nutr Diet 52, 132-138.

10. Scully M, Dixon H, White V et al. (2007) Dietary, physical activity and sedentary behaviour among Australian secondary students in 2005. Health Promot Int 22, 236-245.

11. Campbell KJ, Crawford DA, Salmon J et al. (2007) Associations between the home food environment and obesitypromoting eating behaviors in adolescence. Obesity (Silver Spring) 15, 719-730.

12. Field AE, Austin SB, Gillman MW et al. (2004) Snack food intake does not predict weight change among children and adolescents. Int J Obes Relat Metab Disord 28, 1210-1216.

13. Wardle J, Jarvis MJ, Steggles N et al. (2003) Socioeconomic disparities in cancer-risk behaviors in adolescence: baseline results from the Health and Behaviour in Teenagers Study (HABITS). Prev Med 36, 721-730.

14. Persson Osowski C, Fjellström C, Olsson U et al. (2012) Agreement between child and parent reports of 10- to 12-year-old children's meal pattern and intake of snack foods. J Hum Nutr Diet 25, 50-58.

15. Commonwealth Scientific Industrial Research Organisation, Preventative Health National Research Flagship, University of South Australia (2008) 2007 Australian National
Children's Nutrition and Physical Activity Survey - Main Findings. Canberra: Commonwealth of Australia.

16. Cameron AJ, Ball K, Pearson N et al. (2012) Socioeconomic variation in diet and activity-related behaviours of Australian children and adolescents aged 2-16 years. Pediatr Obes $\mathbf{7}$, 329-342.

17. Larson N \& Story M (2013) A review of snacking patterns among children and adolescents: what are the implications of snacking for weight status? Child Obes 9, 104-115.

18. Macdiarmid J, Loe J, Craig LC et al. (2009) Meal and snacking patterns of school-aged children in Scotland. Eur J Clin Nutr 63, 1297-1304.

19. Ruxton CH, Kirk TR, Belton NR et al. (1996) Relationships between social class, nutrient intake and dietary patterns in Edinburgh schoolchildren. Int J Food Sci Nutr 47, 341-349.

20. Patrick H \& Nicklas TA (2005) A review of family and social determinants of children's eating patterns and diet quality. J Am Coll Nutr 24, 83-92.

21. Crawford PB, Obarzanek E, Schreiber GB et al. (1995) The effects of race, household income, and parental education on nutrient intakes of 9- and 10-year-old girls. NHLBI Growth and Health Study. Ann Epidemiol 5, 360-368.

22. Hardy L, King L, Espinel P et al. (2011) NSW Schools Physical Activity and Nutrition Survey (SPANS) 2010: Full Report. Sydney: NSW Ministry of Health.

23. Snoek HM, van Strien T, Janssens JMAM et al. (2006) The effect of television viewing on adolescents' snacking: individual differences explained by external, restrained and emotional eating. J Adolesc Health 39, 448-451.

24. Utter J, Scragg R \& Schaaf D (2006) Associations between television viewing and consumption of commonly advertised foods among New Zealand children and young adolescents. Public Health Nutr 9, 606-612.

25. Phillips SM, Bandini LG, Naumova EN et al. (2004) Energydense snack food intake in adolescence: longitudinal relationship to weight and fatness. Obes Res 12, 461-472.

26. Vereecken CA, Todd J, Roberts C et al. (2006) Television viewing behaviour and associations with food habits in different countries. Public Health Nutr 9, 244-250.

27. Francis LA, Lee Y \& Birch LL (2003) Parental weight status and girls' television viewing, snacking, and body mass indexes. Obes Res 11, 143-151.

28. Ouwens MA, Cebolla A \& van Strien T (2012) Eating style, television viewing and snacking in pre-adolescent children. Nutr Hosp 27, 1072-1078.

29. Pearson N \& Biddle SJH (2011) Sedentary behavior and dietary intake in children, adolescents, and adults. A systematic review. Am J Prev Med 41, 178-188.

30. Pearson N, Ball K \& Crawford D (2011) Mediators of longitudinal associations between television viewing and eating behaviours in adolescents. Int J Behav Nutr Phys Act 8, 23.

31. Lazzeri G, Pammolli A, Azzolini E et al. (2013) Association between fruits and vegetables intake and frequency of breakfast and snacks consumption: a cross-sectional study. Nutr J 12, 123.

32. Sebastian RS, Cleveland LE \& Goldman JD (2008) Effect of snacking frequency on adolescents' dietary intakes and meeting national recommendations. $J$ Adolesc Health $\mathbf{4 2}$, 503-511.

33. Weiss A, Xu F, Storfer-Isser A et al. (2010) The association of sleep duration with adolescents' fat and carbohydrate consumption. Sleep 33, 1201-1209.

34. Heath G, Roach GD, Dorrian J et al. (2012) The effect of sleep restriction on snacking behaviour during a week of simulated shiftwork. Accid Anal Prev 45, Suppl., 62-67.

35. Kim S, DeRoo LA \& Sandler DP (2011) Eating patterns and nutritional characteristics associated with sleep duration. Public Health Nutr 14, 889-895. 
36. Nedeltcheva AV, Kilkus JM, Imperial J et al. (2009) Sleep curtailment is accompanied by increased intake of calories from snacks. Am J Clin Nutr 89, 126-133.

37. Martens M, Van Assema P \& Brug J (2005) Why do adolescents eat what they eat? Personal and social environmental predictors of fruit, snack and breakfast consumption among 12-14-year-old Dutch students. Public Health Nutr 8, 1258-1265.

38. MacFarlane A, Crawford D, Ball K et al. (2007) Adolescent home food environments and socioeconomic position. Asia Pac J Clin Nutr 16, 748-756.

39. Pearson N, Ball K \& Crawford D (2011) Predictors of changes in adolescents' consumption of fruits, vegetables and energy-dense snacks. Br J Nutr 105, 795-803.

40. Cutler GJ, Flood A, Hannan P et al. (2011) Multiple sociodemographic and socioenvironmental characteristics are correlated with major patterns of dietary intake in adolescents. J Am Diet Assoc 111, 230-240.

41. O'Dea JA (2003) Why do kids eat healthful food? Perceived benefits of and barriers to healthful eating and physical activity among children and adolescents. J Am Diet Assoc 103, 497-501.

42. Giskes K, Patterson C, Turrell G et al. (2005) Health and nutrition beliefs and perceptions of Brisbane adolescents. Nutr Diet 62, 69-75.

43. Croll JK, Neumark-Sztainer D \& Story M (2001) Healthy eating: what does it mean to adolescents? J Nutr Educ 33, 193-198.

44. Hill L, Casswell S, Maskill C et al. (1998) Fruit and vegetables as adolescent food choices in New Zealand. Health Promot Int 13, 55-65.

45. Morley BC, Scully M, Niven PH et al. (2012) What factors are associated with excess body weight in Australian secondary school students? Med J Aust 196, 189-192.

46. Australian Bureau of Statistics (2008) Information Paper: An Introduction to Socio-Economic Indexes for Areas (SEIFA) 2006. ABS Catalogue no. 2039.0. Canberra: ABS.

47. Department of Primary Industries and Energy, Department of Human Services and Health (1994) Rural, Remote and Metropolitan Areas Classification 1991 Census Edition. Canberra: Commonwealth of Australia.

48. Davies PSW, Roodveldt R \& Marks G (2001) Standard Methods for the Collection and Collation of Anthropometric Data in Children. Canberra: Commonwealth Department of Health and Aged Care.

49. Cole T, Bellizzi M, Flegal K et al. (2000) Establishing a standard definition for child overweight and obesity worldwide: international survey. BMJ 320, 1240-1243.

50. Flood V, Webb K \& Rangan A (2005) Recommendations for Short Questions to Assess Food Consumption in Children for the NSW Health Surveys. Sydney: NSW Centre for Public Health Nutrition.

51. Prochaska JJ, Sallis JF \& Long B (2001) A physical activity screening measure for use with adolescents in primary care. Arch Pediatr Adolesc Med 155, 554-559.

52. Hu FB, Rimm EB, Stampfer MJ et al. (2000) Prospective study of major dietary patterns and risk of coronary heart disease in men. Am J Clin Nutr 72, 912-921.

53. Mishra G, Ball K, Arbuckle J et al. (2002) Dietary patterns of Australian adults and their association with socioeconomic status: results from the 1995 National Nutrition Survey. Eur J Clin Nutr 56, 687-693.

54. Ball K, MacFarlane A, Crawford D et al. (2009) Can social cognitive theory constructs explain socio-economic variations in adolescent eating behaviours? A mediation analysis. Health Educ Res 24, 496-506.

55. National Health and Medical Research Council (2013) Australian Dietary Guidelines. Canberra: NHMRC.

56. Australian Bureau of Statistics (2010) Schools Australia 2009. ABS Catalogue no. 4221.0. Canberra: ABS.

57. Australian Bureau of Statistics (2008) Census of Population and Housing: Socio-Economic Indexes for Areas (SEIFA) Australia - Data only 2006. ABS Catalogue no. 2033.0.55.001. Canberra: ABS.

58. Howe AS, Black KE, Wong JE et al. (2013) Dieting status influences associations between dietary patterns and body composition in adolescents: a cross-sectional study. Nutr J 12, 51.

59. Champagne CM, Baker NB, DeLany JP et al. (1998) Assessment of energy intake underreporting by doubly labeled water and observations on reported nutrient intakes in children. J Am Diet Assoc 98, 426-433.

60. Perks SM, Roemmich JN, Sandow-Pajewski M et al. (2000) Alterations in growth and body composition during puberty. IV. Energy intake estimated by the youthadolescent food-frequency questionnaire: validation by the doubly labeled water method. Am J Clin Nutr $\mathbf{7 2}$, $1455-1460$.

61. Cross AT, Babicz D \& Cushman LF (1994) Snacking patterns among 1,800 adults and children. J Am Diet Assoc 94, 1398-1403.

62. Young K, Kennedy V, Kingsland M et al. (2012) Healthy food and beverages in senior community football club canteens in New South Wales, Australia. Health Promot J Aust 23, 149-152.

63. Kelly B, Chapman K, King L et al. (2008) Double standards for community sports: promoting active lifestyles but unhealthy diets. Health Promot J Aust 19, 226-228.

64. Kelly B, Baur LA, Bauman AE et al. (2010) Examining opportunities for promotion of healthy eating at children's sports clubs. Aust N Z J Public Health 34, 583-588.

65. Kelly B, Bauman AE \& Baur LA (2014) Population estimates of Australian children's exposure to food and beverage sponsorship of sports clubs. J Sci Med Sport 17, 394-398.

66. Cairns G, Angus K \& Hastings G (2009) The Extent, Nature and Effects of Food Promotion to Children: A Review of the Evidence to December 2008. Prepared for the World Health Organization. Geneva: WHO.

67. Livingstone S \& Helsper E (2004) Advertising Foods to Children: Understanding Promotion in the Context of Children's Daily Lives. A review of the literature prepared for the Research Department of the Office of Communications (OFCOM). London: Department of Media and Communications, London School of Economics.

68. Committee on Food Marketing and the Diets of Children and Youth (2006) Food Marketing to Children and Youth: Threat or Opportunity? [J McGinnis, J Appleton Gootman and VI Kraak, editors]. Washington, DC: National Academy of Sciences.

69. Martin K, Rosenberg M, Miller M et al. (2010) Move and Munch Final Report. Trends in Physical Activity, Nutrition and Body Size in Western Australian Children and Adolescents: The Child and Adolescent Physical Activity and Nutrition Survey (CAPANS) 2008. Perth: Western Australian Government.

70. Hardy LL, King L, Espinel P et al. (2011) Methods of the NSW Schools Physical Activity and Nutrition Survey 2010 (SPANS 2010). J Sci Med Sport 14, 390-396. 